\title{
Der Heidelberger Ansatz der Konfliktdatenerfassung
}

Die quantitativ-vergleichende Konfliktforschung hat in der zurückliegenden Dekade bedeutende Fortschritte gemacht. Zum einen hat die technische Innovation zu einer enormen Ausweitung und Vertiefung des Wissens über politische Konflikte geführt. Zum anderen wurden von Seiten der mit der quantitativen Datenerfassung beschäftigten Forschungseinrichtungen große konzeptionelle Anstrengungen unternommen, dieser empirischen Informationsfülle gerecht $z u$ werden und eine realistische Erfassung des zeitgenössischen Konfliktgeschehens zu ermöglichen. Insbesondere die geografische Disaggregation des Konfliktgeschehens und die Hinwendung zu nicht-staatlichen Akteuren haben hier beträchtliche Fortschritte gezeitigt. Dennoch verbleiben Forschungsdesiderata. Dies betrifft insbesondere die Berücksichtigung nicht-und niedrig-gewaltsamer Konflikte und Konfliktphasen, die konzeptionelle Integration unterschiedlicher Konflikttypen in ein einheitliches Modell, die Betrachtung von Konfliktdynamiken sowie die valide Messung der Intensität des Konfliktaustrags. Der Beitrag vergleicht vor diesem Hintergrund den Stand der Grundlagenforschung führender Konfliktdatenbanken miteinander und geht dabei insbesondere auf den neuen, zum Untersuchungsjahr 2011 reformierten Ansatz der Heidelberger Konfliktforschung ein.

Schlagworte: Quantitative Konfliktforschung, Konfliktdatenbanken, Konfliktintensität, georeferenzielle Verortung

\section{Hinführung}

Spätestens seit der Debatte um die sogenannten »neuen Kriege« (Kaldor 1999; Münkler 2002; Brzoska 2004) begann innerhalb der quantitativ-empirischen Konfliktforschung eine Diskussion um eine realitätsnähere Erfassung des tatsächlichen Konfliktgeschehens (Hasenclever 2002; Sambanis 2002; 2004; Schlichte 2002; 2006; Chojnacki 2004; 2006), die bis heute nicht abgeklungen ist (Mello 2010). In der Kritik standen dabei insbesondere die zu einseitige Fokussierung der Forschung auf Kriege und die dadurch bedingte Vernachlässigung von gewaltsamen Konflikten unterhalb der Kriegsschwelle und von gewaltfreien Konflikten, die zu geringe Beachtung von Wandel und Veränderung innerhalb konkreter Konflikte, eine ungenügende Betrachtung nicht-staatlicher Konfliktakteure, die Vernachlässigung von transnationaler Konfliktführung und Auseinandersetzungen auf substaatlicher 
Ebene sowie die verbreiteten »Silo-Lösungen« in der Typologie innerstaatlicher Konflikte: induktiv gewonnene Unterscheidungen und voneinander getrennte Erfassungen innerstaatlicher Konfliktphänomene (wie Revolutionen, terroristische Anschläge, Putsche, Auseinandersetzungen zwischen nicht-staatlichen Akteuren ohne staatliche Beteiligung oder klassische Bürgerkriege), die ein fragmentiertes Bild des Konfliktpanoramas bedingten (Seybolt 2002; Eck 2005; Pavlovic et al. 2008).

In allen diesen Bereichen sind mittlerweile Fortschritte erzielt worden. So wurden Konfliktdatensätze um gewaltsame Konflikte unterhalb der Kriegsschwelle erweitert (Gleditsch et al. 2002; Themnér/Wallensteen 2012) und nicht-staatliche Akteure (Cunningham et al. 2009; Salehyan et al. 2011; Schlichte 2009; Pettersson/Themnér 2012), Konflikte ohne staatliche Beteiligung (Fjelde/Nilsson 2012; Sundberg et al. 2012) sowie geografische Konfliktinformationen aufgenommen (Buhaug/Lujala 2005; Salehyan/Gleditsch 2006; Weidmann 2009; Ó Tuathail 2010; Raleigh 2010; Korf 2011).

Vier zentrale Desiderata verbleiben jedoch: Erstens die quantitativ vergleichende Erfassung von Konflikten und Konfliktphasen ohne oder mit nur geringer Gewaltsamkeit. Erst hierdurch wird es möglich, das empirische Spektrum politischer Konflikte in seiner Breite in den Blick zu nehmen und Konfliktverläufe durchgängig nachzuzeichnen. Zweitens die konzeptionelle Integration unterschiedlicher Konflikttypen in ein einheitliches Modell, um der angesprochenen "Silo-Problematik" entgegenzuwirken. Aus diesen beiden Punkten ergibt sich drittens die Notwendigkeit einer dynamisierten Betrachtung politischer Konflikte, so dass trotz eines etwaigen Wandels konstitutiver Charakteristika der betreffende Konflikt innerhalb eines einzigen Konzeptes und Datensatzes verbleibt. Das vierte Desideratum besteht sodann darin, dass zu einer validen Messung von Konfliktintensitäten eine mehrdimensionale, über eine Erfassung der Zahl der konfliktbedingten Todesopfer hinausgehende Operationalisierung angezeigt ist.

Ein solches Forschungsprogramm stellt die politikwissenschaftliche Konfliktforschung an der Universität Heidelberg zur Verfügung, das die verschiedenen niedrigintensitären Konflikttypen konzeptionell integriert und neue deduktiv hergeleitete Möglichkeiten der Differenzierung bietet. Die Heidelberger Konfliktforschung umfasst die Arbeit zweier am Institut für Politische Wissenschaft der Universität Heidelberg angesiedelten Forschungseinrichtungen: des 1991 gegründeten Heidelberger Instituts für Internationale Konfliktforschung e.V. (HIIK), das bis 1998 auch die eigene Konfliktdatenbank KOSIMO (Konflikt-Simulations-Modell) pflegte (Pfetsch 1991 a; Billing 1992; Pfetsch/Billing 1994; Pfetsch/Rohloff 2000 a; 2000 b), und der seit 2005 bestehenden Forschungsgruppe des Conflict In- 
formation and Analysis System (CONIAS) ${ }^{1}$ (Schwank 2012). Im vorliegenden Beitrag wird die gemeinsame konfliktwissenschaftliche Methode von CONIAS und HIIK vorgestellt und ihr empirischer Mehrwert aufgezeigt.

Die quantitative Konfliktforschung als Grundlagenforschung verfolgt als zentrales Ziel, das Konfliktgeschehen empirisch möglichst genau zu erfassen und der Konfliktursachenforschung in Form von Datensätzen für weitergehende Analysen zur Verfügung zu stellen. Vergleichende Analysen (Eberwein/Chojnacki 2001; Hegre/Sambanis 2006; Dixon 2009) haben jedoch gezeigt, dass die vorhandenen Konfliktdatensätze nur einen geringen gemeinsamen Deckungsgrad aufweisen. Neben Umfang und Zuverlässigkeit der verwendeten Quellen gelten die Unterschiede in der Erhebungsmethodik als wesentlicher Faktor zur Erklärung der beobachteten Diskrepanzen zwischen den verschiedenen Konfliktdatenbanken. Diese betreffen primär die Konzeption und Operationalisierung des Konfliktbegriffs.

In der quantitativen Konfliktforschung lassen sich drei Richtungen der Definition und Operationalisierung des Konflikt- beziehungsweise Kriegsbegriffs unterscheiden (Most/Starr 1983; Schwank 2012):

1.) Offizielle Kriegserklärungen. Dieser Ansatz wurde in den ersten quantitativen Untersuchungen zur Erfassung zwischenstaatlicher Konflikte verwendet (Wright 1942). Inzwischen gilt dieses Verfahren als veraltet, da gegenwärtige Kriege, schon allein aus völkerrechtlichen Gründen, in der Regel nicht mehr »offiziell« erklärt werden.

2.) Anzahl der Kriegsopfer. Diese Methode der quantitativen Operationalisierung findet sich bei den beiden am häufigsten verwendeten Konfliktdatenbanken, dem Correlates of War Project (COW) (Singer/Small 1972) und dem Uppsala Conflict Data Program (UCDP) (Gleditsch et al. 2002). Beide Projekte benennen inzwischen die Schwelle von 1000 durch den jeweiligen Konflikt hervorgerufenen Todesopfern pro Jahr sowie die Beteiligung mindestens eines Staates als wichtigste Kriterien für das Vorhandensein eines Krieges. Um die Vielzahl kleinerer gewaltsamer Konflikte zu erfassen, wurde in den Konfliktdatensätzen des UCDP die Kategorie der »Minor Armed Conflicts« geschaffen. Hier gilt ein Schwellenwert von 25 Todesopfern pro Jahr.

3.) Beschaffenheit des Konfliktaustrags, das heißt die Art und Weise, in der ein Konflikt geführt wird. Der ungarische Friedens- und Konfliktforscher Istvan Kende (1971; 1982) präsentierte einen Ansatz, bei dem die Qualität des Konfliktaustrags den Ausgangspunkt für die Konfliktdefinition und weitere Differenzierungen bildet.

1 Bis Januar 2013 wurde die Datenbank unter dem Namen CONIS (Conflict Information System) geführt. Aus markenrechtlichen Gründen wurde der Name geändert. 
Diesem Ansatz der qualitativen Operationalisierung schlossen sich die in Deutschland beheimateten und unabhängig voneinander Ende der 1980er Jahre begründeten Konfliktdatenbankprojekte der Arbeitsgemeinschaft Kriegsursachenforschung (AKUF) in Hamburg (Gantzel 1986; 1988) und des KOSIMO in Heidelberg (Pfetsch 1991 a; Billing 1992; Pfetsch/Rohloff 2000 b) an.

Sowohl das vom HIIK herausgegebene Konfliktbarometer als auch die CONIASDatenbank stehen somit methodisch in der Tradition der qualitativen Konfliktdefinition und -operationalisierung. Im Unterschied zum AKUF-Ansatz erfasst CONIAS jedoch auch konkrete Maßnahmen und Ereignisse, auf deren Grundlage ein Konflikt erfasst und seine Intensität bestimmt wird (Schwank 2012). Dies zielt darauf, die Nachvollziehbarkeit und Intersubjektivität der Konfliktbestimmungen zu erhöhen. Bis zum Jahr 2011 waren die Konfliktmaßnahmen die zentrale Grundlage zur Bestimmung der Intensität politischer Konflikte.

Ab dem Beobachtungsjahr 2011 wurde das gemeinsame konfliktwissenschaftliche Verfahren von CONIAS und HIIK weiter ausdifferenziert und systematisiert. So erfolgt zum einen die Bestimmung der Intensität eines Konflikts nun nicht mehr nur wie bisher auf der Ebene der Nationalstaaten und Kalenderjahre (vgl. Schwank 2012), sondern auch für die einzelnen politischen Einheiten auf subnationaler Ebene und auf der Grundlage der Kalendermonate. Zum anderen erfolgt die Bestimmung der Konfliktintensität jetzt durch eine Analyse mittels klar konzipierter Indikatoren zur Bewertung der Mittel und Folgen des Konfliktaustrags, die jedoch weiterhin auf dem Handeln und Kommunizieren der Konfliktakteure basiert. Durch diese konzeptionelle Ausdifferenzierung und Standardisierung der Datenbearbeitung wird eine größere Genauigkeit, Nachvollziehbarkeit und Reliabilität der Informationen zu politischen Konflikten erreicht (vgl. die Kritik von Sundberg et al. 2012).

Diese grobe Charakterisierung des Heidelberger Ansatzes wird im Folgenden genauer expliziert. Daran anschließend wird das Vorgehen anhand eines empirischen Beispiels illustriert. In einem dritten Schritt wird der Heidelberger Ansatz mit anderen Ansätzen zur Erfassung des globalen Konfliktgeschehens verglichen. Abschließend werden die Ergebnisse mit Blick auf die eingangs identifizierten Forschungsdesiderata zusammengefasst. 


\section{Der methodische Ansatz}

Im Folgenden wird zunächst das Basiskonzept des politischen Konflikts vorgestellt und sodann die Definition und Operationalisierung der Konfliktintensitäten dargelegt. $^{2}$

\subsection{Das Basiskonzept des politischen Konflikts}

Nach dem Verständnis des Heidelberger Ansatzes besteht das Wesen eines Konflikts in einem Widerspruch, der mit dem Konzept der Positionsdifferenz erfasst wird (Pfetsch 1991 a; 1991 b). Eine Positionsdifferenz ist eine wahrgenommene Inkompatibilität von Denk- und Vorstellungsinhalten (Glasl 2002) zwischen Individuen beziehungsweise sozialen Gruppen. Zentral für dieses Verständnis ist das Vorhandensein von Akteuren, die in Bezug auf bestimmte Themen beziehungsweise Objekte kommunizieren beziehungsweise handeln. Diese Handlungen und Kommunikationen werden im Heidelberger Ansatz mit der Einführung von CONIAS als Maßnahmen bezeichnet (vgl. Schwank 2012). Ihr Bezugspunkt bildet den Gegenstand der Positionsdifferenz. Akteure, Maßnahmen und Gegenstände bilden somit die konstitutiven Attribute eines sozialen Konflikts (siehe Abbildung 1).

Abbildung 1: Das Heidelberger Konfliktkonzept

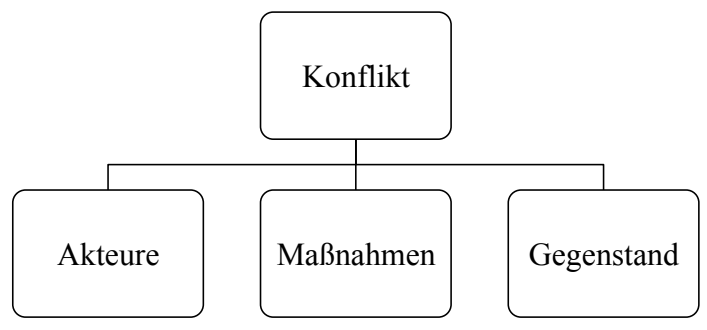

Politische Konflikte bilden einen Subtyp sozialer Konflikte. Durch die weitere Spezifizierung der konstitutiven Attribute werden all jene Formen sozialer Konflikte ausgeschlossen, die nicht als genuin politisch betrachtet werden können. Das »Politische« an politischen Konflikten ergibt sich aus drei notwendigen Bedingungen: Erstens bezieht sich ein politischer Konflikt immer auf einen Gegenstand von gesamtgesellschaftlicher Relevanz, zweitens wird er mittels Maßnahmen durchge-

2 Die Überarbeitung der Heidelberger Methodik erfolgte unter der maßgeblichen Mitwirkung von Stephan Giersdorf, Mark Gombert, Natalie Hoffmann, Jens Hofmann, Lotta Mayer, Gregor Pawlowski und Tobias Selge. 
führt, die außerhalb anerkannter Mechanismen zur Konfliktverregelung liegen, und drittens beteiligen sich an dem Konflikt Akteure, die als durchsetzungsfähig wahrgenommen werden (Schwank et al. 2012). Durch diese Spezifizierung werden beispielsweise Streitigkeiten zwischen Ehepartnern, die meisten Formen von Kriminalität, internationale Handelsstreitigkeiten, viele gesellschaftliche Protestformen, aber auch die "gewöhnliche« Konkurrenz zwischen Regierung und Opposition in Demokratien oder zwischen Teileliten in autokratischen Systemen für sich genommen nicht unter dem Begriff des politischen Konflikts subsumiert.

Als Konfliktakteure gelten jene individuellen oder kollektiven, durch ihre interne Kohäsion nach außen abgrenzbaren Akteure, die als durchsetzungsfähig eingestuft werden können. Ein Akteur gilt dann als durchsetzungsfähig, wenn er von einem anderen Konfliktakteur als solcher wahrgenommen wird und daraus bei diesem eine konfliktbezogene Verhaltensänderung resultiert. Wenn beispielsweise eine extremistische Gruppierung mit ihren Forderungen an die Regierung bei dieser in keiner erkennbaren Weise, und sei sie nur verbal, eine Reaktion hervorruft, gilt diese Gruppierung nicht als durchsetzungsfähig. Die »Durchsetzungsfähigkeit« eines Akteurs wird somit nicht anhand seiner Ausstattung mit Handlungsressourcen, sondern mittelbar auf Basis der Reaktion des Kontrahenten bestimmt.

$\mathrm{Zu}$ den möglichen kollektiven Konfliktakteuren zählen Staaten, internationale Organisationen und nicht-staatliche Akteure. Von den sich hierdurch ergebenden Konstellationen erfasst CONIAS Konflikte zwischen Staaten (zwischenstaatliche Konflikte), Konflikte zwischen einem Staat und einem ihm zugehörigen nichtstaatlichen Akteur (innerstaatliche Konflikte), Konflikte zwischen einem Staat und einem nicht-staatlichen Akteur, der einem anderen Staat zugehörig ist (transnationale Konflikte) sowie Konflikte zwischen nicht-staatlichen Akteuren ohne direkte Staatsbeteiligung (substaatliche Konflikte) (vgl. Schwank/Trinn 2010).

Als Konfliktmaßnahmen werden alle Handlungen und Kommunikationen bezeichnet, die ein Konfliktakteur in einem politischen Konflikt durchführt (vgl. Croissant et al. 2009). Zu unterscheiden sind hierbei, ausdifferenzierter als bisher, konstitutive und korollare Konfliktmaßnahmen.

Konstitutive Konfliktmaßnahmen sind die methodisch primär »relevanten« Maßnahmen: An ihnen wird die Existenz eines politischen Konflikts erkennbar, das heißt erst sie konstituieren (neben den Konfliktakteuren und -gegenständen) eine soziale Konfliktsituation als politischen Konflikt. Eine konstitutive Konfliktmaßnahme liegt vor, wenn die betreffende Handlung oder Kommunikation außerhalb etablierter Regelungsverfahren liegt und wenn sie, gegebenenfalls im Zusammenwirken mit anderen Maßnahmen, eine staatliche Kernfunktion oder die völkerrechtliche Ordnung bedroht oder eine solche Bedrohung in Aussicht stellt. 
Etablierte Regelungsverfahren sind solche Mechanismen des Konfliktmanagements, die von den beteiligten Konfliktakteuren anerkannt werden. Zu ihnen können beispielsweise Wahlen oder Gerichtsverfahren zählen. Ein Regelungsverfahren kann nur dann als anerkannt gelten, wenn es gewaltvermeidend ist, das heißt wenn zu seiner Durchführung keine physische Gewalt angewandt oder angedroht wird. Als staatliche Kernfunktionen werden die Aufrechterhaltung der physischen Sicherheit einer Bevölkerung, der Integrität eines Territoriums und einer politischen, sozioökonomischen oder kulturellen Ordnung verstanden. Eine staatliche Kernfunktion oder die völkerrechtliche Ordnung gilt dann als bedroht, wenn das Fortdauern der Maßnahme(n) die Erfüllung der Funktionen beziehungsweise das Fortbestehen der Ordnung in der Wahrnehmung eines Konfliktakteurs unmöglich oder unwahrscheinlich macht.

Korollare Konfliktmaßnahmen sind nicht ausschlaggebend für die Konstituierung politischer Konflikte, das heißt sie liegen innerhalb etablierter Regelungsverfahren und/oder bedrohen eine staatliche Kernfunktion oder die völkerrechtliche Ordnung nicht. Sie treten gewissermaßen »zusätzlich « zu konstitutiven Konfliktmaßnahmen auf. So hat beispielsweise während des Bürgerkriegs in Sri Lanka zwischen der Regierung und den Befreiungstigern von Tamil Eelam (LTTE) der Premierminister des Landes als Zeichen des guten Willens hinduistische Tempel besucht. Auch wenn solche Handlungen für den Fortgang eines Konflikts entscheidend sein können, ist ihr Auftreten weder notwendig noch hinreichend für die Erfassung eines politischen Konflikts. Wird ein sozialer Konflikt ausschließlich mittels korollarer Maßnahmen ausgetragen, tragen zwei Staaten eine Territorialstreitigkeit beispielsweise durchgehend vor dem Internationalen Gerichtshof aus, wird dieser Konflikt folglich nicht als politischer Konflikt verstanden.

Die empirisch auftretenden konstitutiven und korollaren Maßnahmen werden in CONIAS einzeln erfasst und einer von über siebzig Maßnahmenkategorien zugeordnet. Dadurch wird die breite und differenzierte Erfassung politischer, ökonomischer, juristischer, medialer, militärischer, terroristischer, insurgenter, protestbezogener und repressiver Handlungen und Kommunikationen möglich.

Als Konfliktgegenstände werden diejenigen Güter verstanden, die von den Konfliktakteuren durch konstitutive Konfliktmaßnahmen angestrebt werden und gesamtgesellschaftliche Relevanz besitzen, das heißt einen Bezug zum gesellschaftlichen Zusammenleben innerhalb eines Staates oder zur Koexistenz von Staaten im internationalen System aufweisen. Prinzipiell kann jede materielle oder immaterielle Entität zum Ziel des Wollens von Konfliktakteuren werden. Gesamtgesellschaftliche Relevanz ist einem Konfliktgegenstand nicht per se inhärent, sondern ergibt sich erst aus der Art und Weise, mit der das präferierte Ziel verfolgt wird. Ein 
Gut erlangt gesamtgesellschaftliche Relevanz erst dann, wenn es mittels solcher Konfliktmaßnahmen angestrebt wird, die außerhalb etablierter Regelungsverfahren liegen und eine staatliche Kernfunktion oder die völkerrechtliche Ordnung bedrohen oder eine solche Bedrohung in Aussicht stellen.

CONIAS und HIIK klassifizieren die konkret in einem Konflikt auftretenden Gegenstände anhand von zehn Typen, die häufige Ziele von Konfliktakteuren spezifizieren (vgl. Croissant et al. 2009). Zu nennen sind hier erstens nationale Macht, das heißt das Streben nach politischer Herrschaftsgewalt in einem Staat; zweitens Autonomie, worunter die Erlangung oder Ausweitung der politischen Selbstbestimmung einer Bevölkerung in einem Staat (oder in einem abhängigen Gebiet ohne Unabhängigkeitsbestrebungen) verstanden wird; drittens Sezession, also die intendierte Abtrennung eines Teils eines Staatsgebiets mit dem Ziel der Errichtung eines neuen Staates oder des Anschlusses an einen bestehenden Staat. Hiervon ist zu unterscheiden, viertens, Dekolonisierung, das heißt das Ziel der Unabhängigkeit eines abhängigen Gebiets. Neben diese streng an politischer Herrschaft orientierten Konfliktgegenstände tritt fünftens die subnationale Vorherrschaft, also die de facto-Kontrolle einer Regierung, einer nicht-staatlichen Organisation oder einer Bevölkerung über ein Gebiet oder eine Bevölkerung. Diese Konfliktgegenstandstypen kommen lediglich in innerstaatlichen, substaatlichen und transnationalen Konflikten vor. Nur in zwischenstaatlichen Konflikten vergeben werden, sechstens, Territorium, das heißt die Veränderung des Verlaufs einer zwischenstaatlichen Grenze, sowie siebtens internationale Macht, also eine angestrebte Veränderung der Machtkonstellation im internationalen System oder in einem seiner Regionalsysteme. In jedem Konflikttyp auftreten können, achtens, Ressourcen, das heißt der Besitz natürlicher Ressourcen oder Rohstoffe beziehungsweise des hieraus erzielten Profits, sowie, neuntens, Ideologie/System, also eine angestrebte Veränderung der ideologischen, religiösen, sozioökonomischen oder rechtlichen Ausrichtung des politischen Systems oder eine Änderung des Regimetyps. Schließlich ist eine Residualkategorie vorgesehen, mit deren Hilfe selten auftretende Konfliktgegenstände erfasst werden können. Neun der zehn Kategorien werden seit Beginn der Heidelberger Konfliktforschung verwendet; der Typ »subnationale Vorherrschaft « wurde mit CONIAS neu in die Heidelberger Methodik integriert. ${ }^{3}$

Die Heidelberger Konfliktmethodik beruht insgesamt gesehen auf einem möglichst grundlegenden Konzept von politischem Konflikt. Die vorgestellten Dimensionen sind zusammengenommen die notwendigen und hinreichenden Bedingungen

3 In den Konfliktbarometern 2003 bis 2010 des HIIK wurde dieser Konfliktgegenstand als »regionale Vorherrschaft« bzw. »regional predominance« bezeichnet. 
für das Auftreten eines politischen Konflikts, das heißt nur wenn alle drei Definitionskriterien erfüllt sind, wird eine Konfliktsituation als politischer Konflikt eingestuft. Das Konzept ist mithin so spezifisch, dass CONIAS nicht jede denkbare konfliktive Situation erfasst. Zugleich ist aufgrund des hohen Abstraktionsniveaus des Konzepts sein Geltungsbereich so weit, dass alle in der Forschung als relevant diskutierten Formen des politischen Konflikts berücksichtigt werden. Dies vermeidet die eingangs kritisierte »Silo-Lösung« der Konflikttypologie. Hierdurch werden konzeptionelle Kurzschlüsse vermieden, wie zum Beispiel »ethnischer Konflikt« oder »Terrorismus«. Der Heidelberger Ansatz erlaubt demgegenüber durch die skizzierte Spezifizierung der genannten drei Dimensionen eine systematische Typenbildung für die Gesamtheit der politischen Konflikte. So kann beispielsweise der bereits erwähnte Konflikt zwischen der sri-lankischen Regierung und den LTTE, den drei konzeptionellen Dimensionen Akteur, Gegenstand und Maßnahmen folgend, als innerstaatlicher Sezessionskonflikt mit einer Mischung aus konventioneller und terroristischer Gewaltanwendung eingeordnet werden.

Das spezifisch Neue an einer solchen Konzeptualisierung politischer Konflikte ist dabei insbesondere die systematische Inklusion von Konfliktmaßnahmen. Die Ausdifferenzierung der Handlungen von Konfliktakteuren in gewaltsame und gewaltlose Aktivitäten ermöglicht die konzeptionelle Erfassung nicht-, gering- und hochgewaltsamer Konflikte. Der kommunikative Charakter der Konfliktmaßnahmen stellt den Netzwerkcharakter der Akteure in einem Konflikt in den Vordergrund. Zusammen mit der geografischen Ausdehnung des Konfliktaustrags erlaubt dies die Bestimmung der »Größe« eines Konflikts. Außerdem erlauben die Maßnahmen die Bildung von Konfliktphasen. Sie sind damit eine Voraussetzung für die Bestimmung und Analyse von Konfliktdynamiken, die im Folgenden dargestellt werden.

\subsection{Konzept und Operationalisierung der Konfliktintensität}

In der quantitativ vergleichenden Konfliktforschung wird immer wieder der Bedarf an wissenschaftlichen Modellen zur Abbildung von Konfliktdynamiken betont (Bremer 1993; DeRouen/Sobek 2004; DeRouen/Bercovitch 2008). Möglichst vollständige Daten zu Entwicklungswegen von Konflikten sollen helfen, Eskalationsfaktoren politischer Konflikte zu bestimmen. So kann die spezifische Interaktion der Akteure in einem Konflikt selbst kausale Wirkung entfalten und zu einer Eskalation oder Deeskalation eines Konflikts beitragen (Fürnkranz et al. 1997; Schwank 2012). 
Die Heidelberger Konfliktmethodik legt daher besonderen Wert auf die Operationalisierung verschiedener Intensitätsstufen. Die Konfliktintensität kann verstanden werden als eine Eigenschaft einer Gesamtheit von Konfliktmaßnahmen in einem geografischen und zeitlichen Raum. Als primäre zeitliche und räumliche Untersuchungseinheiten werden dabei die Kalendermonate und die Regionen, das heißt die subnationalen Verwaltungseinheiten der ersten Ebene (beispielsweise die Bundesländer in Deutschland oder die Provinzen in Thailand), herangezogen. ${ }^{4}$ Die elementare Konfliktintensität wird somit jeweils für einen »Regionalmonat« ermittelt.

Insgesamt unterscheidet der Ansatz fünf Intensitätsstufen: Disput, gewaltlose Krise, gewaltsame Krise, begrenzter Krieg und Krieg (vgl. Wagschal et al. 2010; HIIK 2012; Schwank 2012; Schwank et al. 2012). Die gewaltsame Krise, der begrenzte Krieg und der Krieg bilden zusammen die Kategorie der Gewaltkonflikte, im Unterschied zu den gewaltfreien Konflikten, also dem Disput und der gewaltlosen Krise (siehe Tabelle 1).

Tabelle 1: Stufen der Konfliktintensität

\begin{tabular}{|l|l|l|}
\hline $\mathbf{1}$ & Disput & \multirow{2}{*}{ gewaltfreie Konflikte } \\
\hline $\mathbf{2}$ & gewaltlose Krise & \\
\cline { 1 - 2 } $\mathbf{3}$ & gewaltsame Krise & \multirow{2}{*}{ Gewaltkonflikte } \\
\cline { 1 - 2 } $\mathbf{5}$ & begrenzter Krieg & \\
\hline
\end{tabular}

Ein Disput ist ein völlig gewaltfrei ausgetragener politischer Konflikt. Obwohl die hierbei auftretenden konstitutiven Konfliktmaßnahmen ohne Einsatz physischer Gewalt erfolgen, müssen sie die Bedrohung staatlicher Kernfunktionen oder der völkerrechtlichen Ordnung zumindest in Aussicht stellen - andernfalls wäre kein politischer Konflikt gegeben. In einer gewaltlosen Krise wird mit der Anwendung von Gewalt gedroht. Neben der expliziten Drohung mit physischer Gewalt zählt hierzu auch die Anwendung von Gewalt gegen Sachen, ohne die physische Verletzung von Personen billigend in Kauf zu nehmen (beispielsweise Sachbeschädigung im Rahmen einer Demonstration), die Weigerung eines Akteurs, seine Waffen abzugeben, obwohl er hierzu von einem anderen Konfliktakteur aufgefordert wurde, das Aufeinanderrichten von Waffensystemen (zum Beispiel Raketen) sowie die Durchführung von Sanktionen.

4 Die von CONIAS herangezogene Liste der subnationalen Verwaltungseinheiten beruht auf dem Standard nach ISO 3166-2. 
Abbildung 2: Zusammenführung der Einzelindikatoren zur Intensität eines Gewaltkonflikts

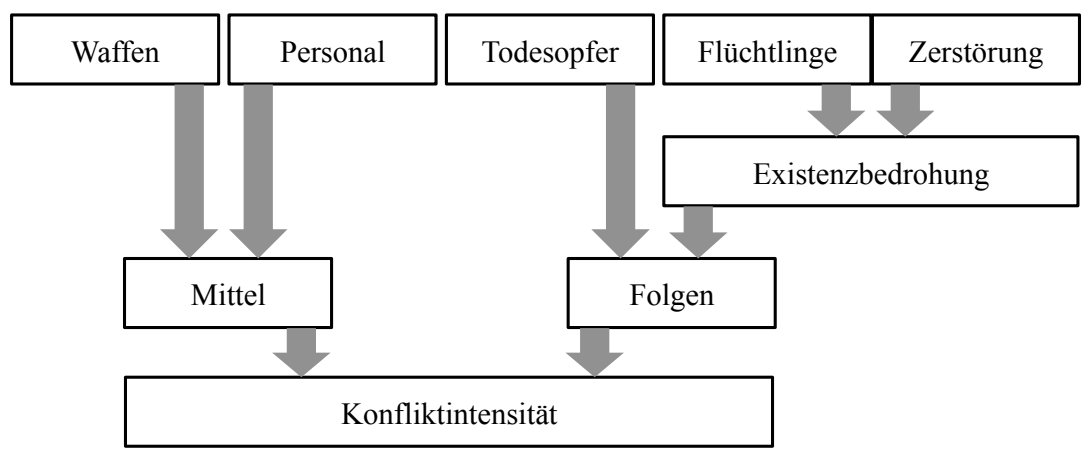

Zur Operationalisierung der drei Stufen des Gewaltkonflikts wurden bislang die Konfliktmaßnahmen innerhalb eines frei bestimmbaren Handlungszusammenhangs als Bewertungsgrundlage verwendet. Seit 2011 werden in einem geregelten und durch eine Dateneingabemaske unterstützen Verfahren insgesamt fünf Indikatoren herangezogen, die die eingesetzten Mittel und die Folgen des gewaltsamen Konfliktaustrags messen. Die Dimension der Mittel umfasst die Indikatoren Waffenund Personaleinsatz, die Dimension der Folgen die Indikatoren Todesopfer, Zerstörung und Flüchtlinge. Für jeden dieser fünf Indikatoren wird auf Basis aller Maßnahmen eines Regionalmonats eine Einschätzung in Form einer Punktzahl gegeben. Zur Bewertung der Gesamtintensität eines Regionalmonats werden die einzelnen Punktwerte stufenweise aggregiert (siehe Abbildung 2).

Zur Bestimmung der gewaltsamen Konfliktintensität wird seit 2011 ein Forschungsprozess durchlaufen, der qualitative und quantitative Forschungsstrategien miteinander kombiniert.

Zunächst wird die Art der eingesetzten Waffen bestimmt. Dabei wird anhand eines Katalogs von Schlüsselwörtern zwischen leichten und schweren Waffen unterschieden sowie hinsichtlich schwerer Waffen die Schwere des Waffeneinsatzes bewertet. Als leichte Waffen gelten Hieb- und Stichwaffen, Bogen- und Wurfwaffen sowie kleine Handfeuer-, Explosions- und Brandwaffen wie Gewehre, Handgranaten und Molotow-Cocktails. Schwere Waffen umfassen demgegenüber schwere Feuerwaffen (zum Beispiel Artilleriegeschütze), atomare, biologische und chemische Kampfstoffe sowie physisch schwere, zumeist panzerbrechende Handfeuer-, Explosions- und Brandwaffen wie schultergestützte Panzerabwehrwaffen, große konventionelle Bomben oder Brandbomben. Ein schwerer Einsatz dieser Waffen liegt vor, wenn sie umfassend genutzt werden, also in massiver Weise oder im Ver- 
bund untereinander. Der Einsatz leichter Waffen wird mit null Punkten bewertet, der leichte Einsatz schwerer Waffen mit einem und der schwere Einsatz schwerer Waffen mit zwei Punkten.

Im Anschluss an diese Bewertung wird die Anzahl der Personen, die in der schwersten Einzelmaßnahme des beobachteten Regionalmonats eingesetzt wurden, bestimmt. Bei dem betrachteten Konfliktpersonal handelt es sich im Regelfall um Soldaten oder Polizisten im Falle staatlicher Akteure und um bewaffnete Anhänger oder Söldner im Falle nicht-staatlicher Akteure. Ausschlaggebend sind all jene Personen, die in ihrer Handlung als Kollektiv einen Konfliktakteur innerhalb einer gewaltsamen Kommunikation repräsentieren. Demgemäß werden auch Demonstranten, die selbst nicht gewaltsam handeln, jedoch als Adressaten an einer gewaltsamen Kommunikation beteiligt sind, als relevantes »eingesetztes Personal« betrachtet. Werden in der betrachteten Maßnahme bis zu 50 Personen eingesetzt, wird dies mit null Punkten bewertet. Für Maßnahmen mit einem Personeneinsatz zwischen 50 und 400 wird ein Punkt vergeben. Bei Maßnahmen mit mehr als 400 Personen werden zwei Punkte notiert. ${ }^{5}$

Hinsichtlich der Gewaltfolgen wird zunächst die Gesamtzahl der Todesopfer betrachtet, die durch den Konflikt in dem untersuchten Regionalmonat hervorgerufen wurden (Schwellenwerte: 20 und 60). Als Todesopfer gelten ausschließlich diejenigen Menschen, die durch direkte Gewaltmaßnahmen getötet wurden oder an den Folgen einer solchen Gewaltmaßnahme gestorben sind. Personen, die erst aufgrund mittelbarer Folgeerscheinungen eines Konflikts sterben, zum Beispiel durch Hunger oder Krankheit, fließen nicht mit in die Betrachtung ein. Selbstmordattentate sind auch dann mindestens als gewaltsame Krise zu bewerten, wenn nur der Attentäter selbst getötet oder verletzt wurde. Auch stellt die Vollstreckung einer Verurteilung zum Tode eine gewaltsame Konfliktmaßnahme dar.

Danach wird unter Berücksichtigung aller individuellen Konfliktmaßnahmen eines Regionalmonats die Gesamtzahl der aufgetretenen Flüchtlinge oder Vertriebenen bestimmt (Schwellenwerte: 1000 und 20.000). Als Flucht wird die durch Konfliktmaßnahmen ausgelöste Migration von Personen verstanden. Eine solche Konfliktmaßnahme kann jede Durchführung oder Androhung einer Gewaltmaßnahme

5 Die Schwellenwerte für die nachfolgenden Indikatoren werden jeweils in Klammern angegeben. Numerisch klaren Schwellenwerten haftet freilich fast immer ein gewisses Maß an Willkür an. Die quantitativen Schwellenwerte, die CONIAS verwendet, knüpfen an Auswertungen der CONIASDatenbank und die über zwanzigjährige Erfahrung des HIIK in der qualitativen Bewertung von Konflikten an. Explizites Ziel bei der Einführung quantitativer Indikatoren war es, die Anschlussfähigkeit der nach der methodischen Umstellung ermittelten Intensitäten an die davor stärker interpretativ erarbeiteten Einschätzungen zu gewährleisten, damit das Verständnis, was beispielsweise als »Krieg« zu gelten hat, möglichst konstant blieb. 
sein. Hierzu zählt die gezielte Vertreibung, ebenso aber auch die mittelbare Vertreibung durch das gezielte Herbeiführen unmenschlicher Lebensbedingungen (zum Beispiel Zerstörung von Äckern oder Tötung humanitärer Helfer). Nicht als Flucht zählt die Emigration aufgrund allgemeiner ökonomischer oder politischer Bedingungen in einer Gesellschaft. In die Betrachtung fließen sowohl diejenigen Personen ein, die internationale Grenzen überqueren, als auch solche, die innerhalb einen Staatsgebiets verbleiben. In die Erhebung für diesen Indikator fließen ausschließlich Bewegungs-, nicht aber Bestandsdaten ein. Flüchtlinge, die sich zu einem Zeitpunkt bereits in diesem Status befinden, werden daher nicht mit in die Bewertung der jeweiligen Regionalmonatsintensität einbezogen, sondern nur jene Personen, die in einem gegebenen Monat aktiv auf der Flucht sind. Flüchtlinge, die mehrmals fliehen und zurückkehren, werden jeweils nur einmal erfasst.

Schließlich wird das aus den Konfliktmaßnahmen resultierende Ausmaß der Zerstörung in vier Dimensionen bewertet, die als essenziell für die Zivilbevölkerung betrachtet werden. Dies sind Infrastruktur (zum Beispiel Straßen, Schienen oder Rundfunkmasten) und Wohnraum sowie wirtschaftliche (beispielsweise Fabriken oder Felder) und kulturelle Strukturen (zum Beispiel Kirchen, Bibliotheken oder Kriegsdenkmäler). War das zerstörte Objekt wesentlich für die Funktion der jeweiligen Dimension, gilt die Zerstörung als massiv. Ist in keiner der Dimensionen massive Zerstörung aufgetreten, werden null Punkte vergeben. Bei massiver Zerstörung in bis zu zwei Dimensionen wird ein Punkt notiert, darüber hinaus zwei Punkte.

Zur Bestimmung der Intensität eines Konflikts wie auch zur Erfassung der jeweiligen Akteure, Maßnahmen und Gegenstände werden ausschließlich öffentlich zugängliche Informationsquellen genutzt. Diese umfassen neben wissenschaftlichen Abhandlungen zu Einzelkonflikten insbesondere die internationalen Nachrichtendienste und die großen englisch-, französisch-, spanisch- und deutschsprachigen Zeitungen und Fernsehsender sowie die jeweilige lokal erscheinende Presse. Die Nutzung internationaler wie lokaler Medien kann zu Verzerrungen führen, zum Beispiel wenn das internationale Medieninteresse auf bestimmte, häufig hochgewaltsame, politische Konflikte gerichtet ist und dabei andere, gerade gewaltfrei ausgetragene, Konflikte vernachlässigt oder wenn die Berichterstattung lokaler, womöglich von staatlichen oder nicht-staatlichen Akteuren kontrollierter, Medien einseitig zugunsten oder zuungunsten einer Konfliktpartei gefärbt ist oder Nachrichten über bestimmte politische Konflikte ausblendet oder in den Vordergrund rückt. Die Problematik einer nicht völlig von Einzelinteressen unabhängigen Berichterstattung ist stets gegeben und kann nur durch eine gesonderte Evaluierung der Medien selbst verringert werden. Dies setzt zum einen länderspezifische Kenntnisse der Konfliktbearbeiter voraus und wird ergänzt durch eine von der Leitungs- 
ebene von CONIAS geführte Positivliste möglichst unabhängiger internationaler und lokaler Nachrichtenquellen. Zum anderen macht die Datenbank durch die Speicherung der Quelle jeder einzelnen erfassten Konfliktinformation die darauf aufbauenden Einschätzungen nachvollziehbar.

Von der Objektivitätsproblematik ist die Datenverfügbarkeit an sich zu unterscheiden. Der CONIAS-Ansatz der Konfliktkonzeption, Maßnahmenerfassung und Intensitätsermittlung setzt ein vergleichsweise hohes Informationsniveau voraus. Auch hier kann es zu Verzerrungen kommen, sowohl in geografischer Hinsicht wenn zum Beispiel unterentwickelte oder schwer zugängliche Regionen in den Medien unterrepräsentiert werden - als auch insbesondere in zeitlicher Hinsicht. Erst mit der fortgeschrittenen Verbreitung des Internets etwa ab dem Jahr 2000 kann davon ausgegangen werden, dass das Informationsangebot zu gewaltsamen und nicht-gewaltsamen Konfliktsituationen den Anforderungen des CONIAS-Ansatzes entspricht. ${ }^{6}$

Insbesondere bei der Bewertung der Qualität des Waffeneinsatzes und der konfliktbedingten Zerstörung ist eine subjektive Einschätzung der vorliegenden Informationen durch den jeweiligen Codierer notwendig. Dies betrifft in gewissem Maße auch die grundsätzlich quantitativen Indikatoren zu Personaleinsatz, Todesopfern und Flüchtlingen: Zum einen ist stets die Verlässlichkeit von Zahlenangaben abzuwägen; zum anderen liegen mitunter für einen Regionalmonat keine quantitativen Angaben vor. In diesem Fall greift die Bewertung auf einen Katalog quantifizierender Schlüsselwörter (beispielsweise »tausende«, »dutzende«) zurück, die an die quantitativen Schwellenwerte anknüpfen. Zur Kontrolle der Reliabilität der Codierungen werden die Konfliktverläufe in regional organisierten Arbeitsgruppen diskutiert und die erfassten Informationen in einem dreistufigen Verfahren von erfahrenen Mitarbeitern, wissenschaftlich geschultem Leitungspersonal und externen Regionalexperten begutachtet. Dies betrifft nicht nur die fünf Indikatoren zur Ermittlung der gewaltsamen Konfliktintensitäten, sondern auch die Einschätzung nicht-gewaltsamer Konfliktintensitäten (Wann liegt eine Gewaltandrohung vor?) und insbesondere auch die grundlegenden Kriterien Konfliktakteure, -maßnahmen und -gegenstände, welche bestimmen, wann ein politischer Konflikt vorliegt (Wann

6 Die sich mit der Verwendung öffentlicher Informationsquellen ergebenden Problematiken der Objektivität und Verfügbarkeit von Konfliktdaten ist aus Heidelberger Perspektive nicht durch den Einsatz von Personal vor Ort zu kompensieren. Auch in Konfliktgebieten Anwesende haben zumeist keinen unmittelbaren Zugang zum Konfliktgeschehen, so dass sich auch hier Einschränkungen der Informationen ergeben und sich das Problem der Unabhängigkeit unter Umständen noch verstärken kann. 
wird ein Akteur als durchsetzungsfähig wahrgenommen? Wann ist eine staatliche Kernfunktion bedroht?).

Die ebenfalls seit 2011 erfolgende geografische Disaggregation in CONIAS bezieht sich, wie bereits oben erwähnt, auf die subnationalen Verwaltungseinheiten der ersten Ebene. Dies unterscheidet diese Datenbank von der georeferenziellen Verortung beim Georeferenced Event Dataset (GED) von UCDP (Sundberg et al. 2011; Croicu/Sundberg 2012) und dem Armed Conflict Location and Event Dataset (ACLED) des Peace Research Institute Oslo (PRIO) (Raleigh et al. 2010). Sowohl GED als auch ACLED erfassen Konfliktereignisse punktgenau. GED konstruiert darüber hinaus aus diesen Daten Polygone, die Konfliktgebiete darstellen (Croicu/Sundberg 2012).

Neben der Konstruktion von Konflikträumen anhand von Ereigniscodierungen entweder durch Polygone oder wie von Halvard Buhaug (2005) beschrieben durch die Festlegung von Konfliktzirkeln, die alle Ereignisse eines Konflikts umfassen gibt es die Möglichkeit, die Karte der Erdoberfläche mit einem Raster zu versehen und in diesen fixen Konfliktzellen (vgl. Buhaug 2005) entweder Ereignisse zu verorten oder für diese Zellen Intensitäten zu ermitteln.

Sowohl ACLED als auch GED ordnen die verorteten Ereignisdaten zusätzlich primären und sekundären Verwaltungseinheiten zu. Dies dient jedoch der Lokalisierung der Einzelereignisse und nicht der Bestimmung der Konfliktintensität in einem subnationalen Gebiet. Für GED und ACLED ist die Zuordnung eines Konfliktereignisses zu einer Einheit der politischen Geografie zweitrangig. Für CONIAS ist die Anbindung der Daten an diese Einheiten hingegen von erstrangiger Bedeutung. Die der Intensitätsermittlung zugrundeliegenden fünf Indikatoren sind in ihrer Konzipierung ganz auf diese Verwaltungseinheiten ausgerichtet. Auch wenn Nachteile wie die unterschiedliche Größe von subnationalen Verwaltungseinheiten nicht zu vernachlässigen sind, haben subnationale Verwaltungseinheiten jedoch als geografische Bezugseinheit gegenüber Konfliktzellen den Vorteil, dass zum einen Nachrichtenquellen Konfliktinformationen in erster Linie in diesen konkreten räumlichen Kontexten verorten und zum anderen empirische Daten zu sozioökonomischen, demografischen, politischen und naturgeografischen Erklärungsfaktoren auf der Ebene unterhalb des Nationalstaats vor allem für diese Einheiten vorliegen.

Wir illustrieren nachfolgend zunächst den Heidelberger Ansatz an einem konkreten Beispiel, den Konflikten in Sri Lanka, und demonstrieren sodann den empirischen Mehrwert des Ansatzes an einem Vergleich der CONIAS-Datenbank mit den für die Forschung zentralen quantitativen Konfliktdatenbanken von COW, UCDP und PRIO. 


\section{Empirische Illustration des methodischen Ansatzes}

CONIAS kennt nicht den Sri-Lanka-Konflikt, sondern unterscheidet insgesamt zwölf Einzelkonflikte seit 1945: fünf innerstaatliche im engeren Sinne, fünf substaatliche, einen zwischenstaatlichen sowie einen transnationalen Konflikt, bei denen die Struktur und Dynamik hinsichtlich der Akteure, Gegenstände, Maßnahmen und Intensitäten je Konflikt gesondert erfasst werden. ${ }^{7}$ Das Konfliktpanorama Sri Lankas umfasst einerseits sowohl fast rein gewaltfrei ausgetragene Konflikte (wie zum Beispiel den Konflikt zwischen der muslimischen Bevölkerung und der Regierung des Landes) als auch sich über Jahre erstreckende Bürgerkriege (hier vorrangig der Konflikt zwischen der Regierung Sri Lankas und den separatistischen LTTE). Der Konfliktaustrag in Sri Lanka weist andererseits sowohl Konflikte zwischen Regierung und nicht-staatlichen Akteuren auf (beispielsweise mit unterschiedlichen tamilischen Parteien) als auch weitgehend ohne Staatsbeteiligung geführte substaatliche Konflikte (zum Beispiel zwischen den LTTE und der »LTTEOst«, einer im Jahr 2004 aufgetretenen Splittergruppe).

Die nachfolgenden Abbildungen illustrieren die Möglichkeiten, die eine zeitlich und räumlich disaggregierte Ermittlung der verschiedenen Konfliktintensitäten für die Darstellung und die Analyse politischer Konflikte bietet. Abbildung 3 zeigt einen Ausschnitt des Verlaufs des Konflikts zwischen der Regierung Sri Lankas und den LTTE. Dieser Konflikt begann mit der Gründung der LTTE im Jahr 1976 und eskalierte erstmals im Jahr 1983 zum Krieg. Auf Ebene der Regionalmonatsintensitäten ist der Konflikt in CONIAS für die Jahre zwischen Januar 2000 und Mai 2009 - dem Monat, in dem dieser Konflikt endete - erfasst.

Erkennbar wird zum einen die Schwankung der Intensitäten zwischen den Jahren. So erreichte der Konflikt in den Jahren 2002 bis einschließlich 2005, bedingt durch den von Norwegen vermittelten und überwachten Waffenstillstand zwischen den LTTE und der sri-lankischen Regierung, in dessen Folge die LTTE ihre Forderungen nach einem eigenen Staat zu Autonomieforderungen abschwächte, als Höchstintensität lediglich das Niveau einer gewaltsamen Krise (Stufe 3). Zwischen 2006 bis zum militärischen Sieg über die LTTE im Mai 2009 wurde der Konflikt als vollumfänglicher Krieg ausgetragen (Stufe 5). Die Abbildung zeigt zum anderen

7 Sri Lanka (Muslime), seit 1948; Sri Lanka (Singhalesische Nationalisten) [mit JVP und JHU], seit 1948; Sri Lanka (Hochland-Tamilen), seit 1948; Sri Lanka (Tamilen - Muslime), seit 1948; Sri Lanka (JVP), 1964-1989; Sri Lanka (verschiedene Tamilen-Gruppen) [mit EPRLF, EROS, PLOTE, TELO, TNT, TULF], seit 1972; Sri Lanka (LTTE/Nord- und Ost-Sri Lanka), 1976-2009; Sri Lanka (LTTE - verschiedene Tamilen-Gruppen) [mit EPDP, EPRLF, PLOTE, TELO], 1976-2009; Sri Lanka (Jamiyathul Ulama - Sufis), 1978-2006; India (LTTE), 1987-2009; Sri Lanka - India (LTTE), 1989-1991; Sri Lanka (TMVP - LTTE) [TMVP zuvor als LTTE Karuna], 2004-2009. 
Abbildung 3: Monatsgenauer Verlauf des Sri-Lanka-Konflikts (LTTE/Nord und Ost-Sri Lanka), Januar 2000 bis Mai 2009

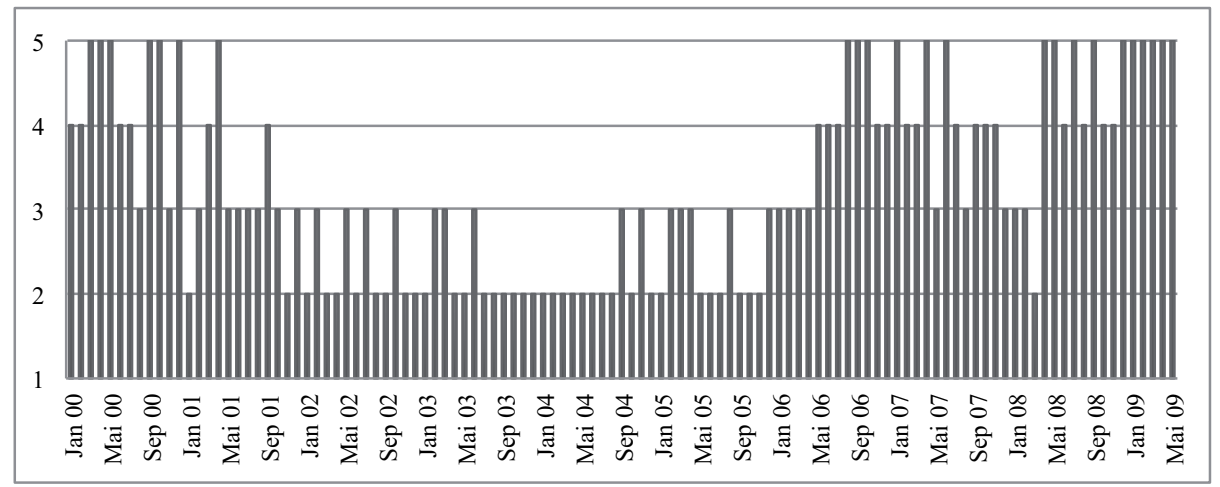

aber auch starke Unterschiede zwischen einzelnen Monaten. So schwankte der Konflikt im Jahr 2000 zwischen einem begrenzten Krieg (Stufe 4) im Januar und Februar, wurde von März bis Mai als Krieg geführt und im Juni und Juli wiederum als begrenzter Krieg. August und November stellten niedrigintensitäre Konfliktphasen dar, in denen der Konflikt maximal als gewaltsame Krise ausgetragen wurde, während er im September, Oktober und Dezember wieder als vollumfänglicher Krieg verlief.

Die Möglichkeiten, die eine mehrdimensionale Operationalisierung disaggregierter Konfliktintensitäten eröffnet, stellt die Abbildung 4 dar. Gezeigt werden für den LTTE-Konflikt die sich aus der Nachrichtenlage ergebenden Punktwerte der fünf der Intensitätsermittlung zugrundeliegenden Indikatoren Waffeneinsatz, Personaleinsatz, Zahl der Todesopfer, Zahl der neu aufgetretenen Flüchtlinge und Ausmaß der Zerstörung bezüglich der Monate August und September 2006 in der Ostprovinz. ${ }^{8}$ Obwohl beide Regionalmonatsintensitäten die Höchststufe 5 (Krieg) aufweisen, lassen sich hinsichtlich der Dimensionen, auf denen diese Einschätzung beruht, deutliche Unterschiede erkennen. Es ergibt sich auf diese Weise gewissermaßen ein hochgradig disaggregiertes »Profil« des Konfliktaustrags.

8 In Sri Lanka stellen die Provinzen die subnationalen Verwaltungseinheiten der ersten Ebene dar. Sie dienen daher wie oben beschrieben als die Untersuchungseinheiten für die Intensitätsermittlung. 
Abbildung 4: Profil des Konfliktaustrags nach Intensitätsindikatoren für den Sri-LankaKonflikt (LTTE/Nord- und Ost-Sri Lanka), August und September 2006, Ostprovinz, Intensitätsstufe 5 .

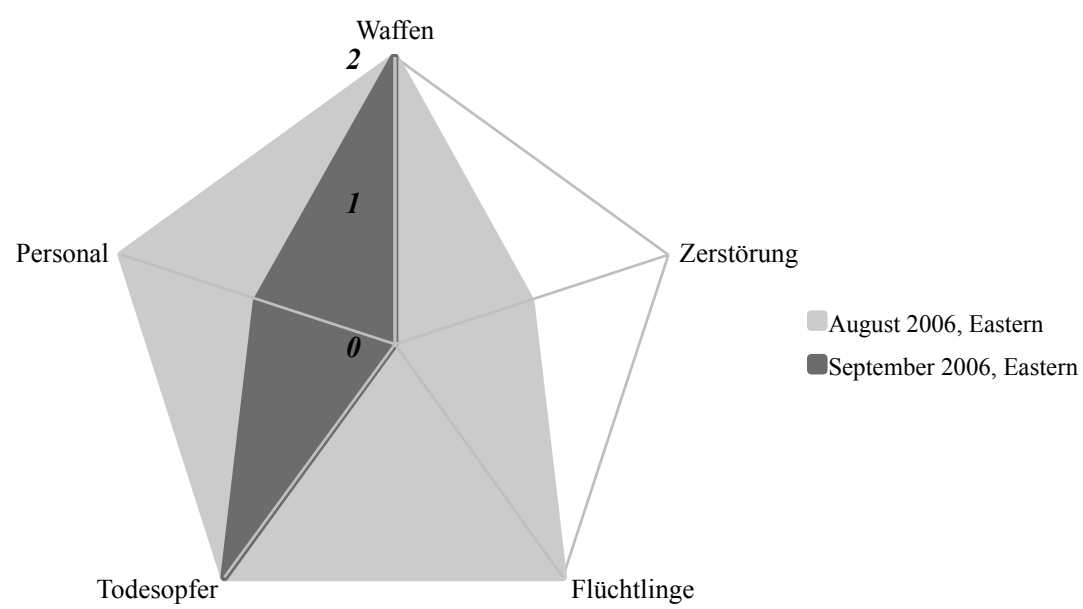

Abbildung 5 zeigt in Kartenform für den selben Konflikt die Regionalmonatsintensitäten des Jahres 2006 in seiner geografischen Ausdehnung. In diesem Jahr verlor das oben erwähnte Waffenstillstandsabkommen endgültig an Bedeutung und der Konflikt eskalierte erneut kriegerisch. Deutlich wird durch die geografische Analyse insbesondere die räumliche Konzentration des gewaltsamen Konfliktaustrags auf bestimmte Provinzen Sri Lankas. Während in Sabaragamuwa und Uva keine und in der Nordwest-, der Süd- und der Westprovinz kaum gewaltsame Maßnahmen in diesem Konflikt ergriffen wurden, waren vor allem - dem hauptsächlichen Siedlungsgebiet der Tamilen in Sri Lanka entsprechend - die Ost- und die Nordprovinz von dem Kriegsgeschehen betroffen. Aber auch in der angrenzenden Nördlichen Zentralprovinz und in der Zentralprovinz, in der die Hauptstadt Sri Lankas gelegen ist, kam es zu Gewalt.

Die monatsweise Abfolge der Konfliktkarten zeigt aber auch hier Unterschiede und Schwankungen der Konfliktintensität. Wurde der Konflikt in der Nordprovinz bereits seit Mai 2006 wieder als begrenzter Krieg geführt, trat die Ostprovinz erst im Juli wieder in eine hochgewaltsame Phase ein. Während der Konflikt dort jedoch bis zum Jahresende durchgängig kriegerisch fortgeführt wurde, erlebte die Nordprovinz im Juli, November und Dezember den Konflikt als gewaltsame Krise, was sich im Übrigen auch im Januar 2007 noch fortsetzte. 
Abbildung 5: Geografische Verteilung der Regionalmonatsintensitäten des Sri-Lanka-Konflikts (LTTE/Nord- und Ost-Sri Lanka), Januar bis Dezember 2006

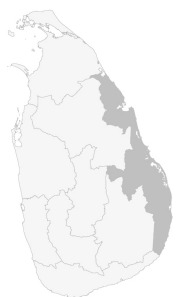

Januar

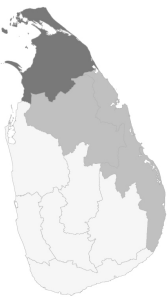

Mai

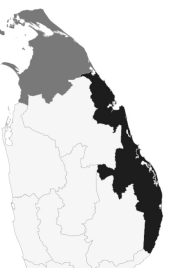

September

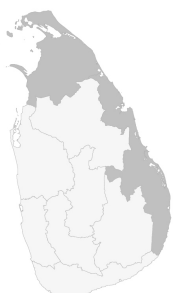

Februar

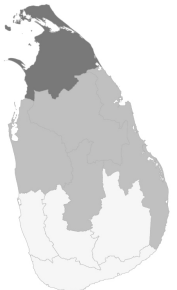

Juni

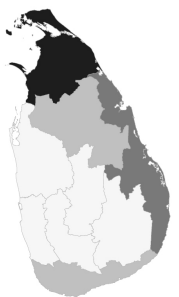

Oktober

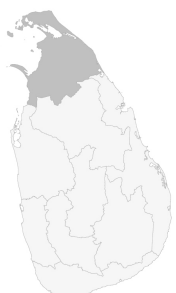

März

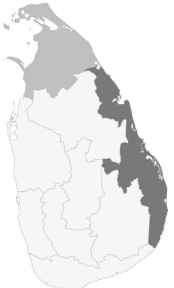

Juli

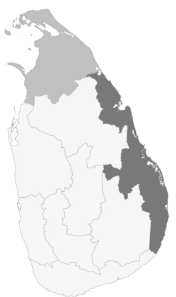

November

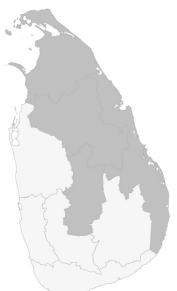

April

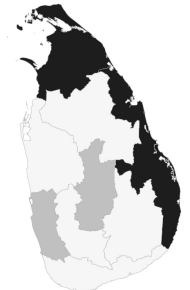

August

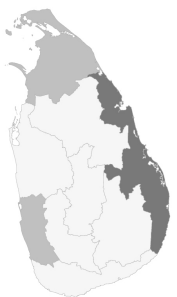

Dezember

kein gewaltsamer Konflikt

Krise

- Begrenzter Krieg

ロ Krieg 


\section{CONIAS im Vergleich mit anderen Konfliktdatenbanken}

Das folgende Kapitel vergleicht systematisch den CONIAS-Datensatz mit den weltweit führenden Konfliktdatenbanken und zieht anschließend anhand der oben identifizierten Forschungsdesiderata ein bewertendes Resümee.

\section{1 Überblick}

Die CONIAS-Datenbank enthält eine global angelegte Erfassung von derzeit 985 Konflikten zwischen 1945 und 2008. ${ }^{9}$ Durchgängig und dynamisch erfasst sind für diese Konflikte ihre jeweiligen Gegenstände (ca. 19.330 Jahresdaten; häufigste Konfliktgegenstände sind Territorium (4566), Ideologie/System (3201), Nationale Macht (2328) und Sezession (2178)), die beteiligten Akteure (ca. 41.612 Jahresdaten; davon $51,6 \%$ staatliche, $0,4 \%$ supra- und internationale Organisationen und 48\% nicht-staatliche Akteure) bzw. Akteurskonstellationen (ca. 25.498 Jahresdaten; davon 32,6\% in der Konstellation Staat vs. Staat, 54,2\% Staat vs. nicht-staatlicher Akteur und 13,2\% nicht-staatlicher Akteur vs. nicht-staatlicher Akteur), die von den Akteuren eingesetzten Maßnahmen (ca. 45.029) sowie die hieraus abgeleiteten Konfliktintensitäten. ${ }^{10}$ Letztere liegen für den gesamten Erfassungszeitraum als Konfliktjahresdaten vor (13.900 Konfliktjahre). Für den Zeitraum von 2000 bis einschließlich 2009 liegen in der CONIAS-Datenbank bezüglich der innerstaatlichen Gewaltkonflikte der Regionen Asien und Ozeanien sowie Subsaharisches Afrika Regionalmonatsintensitäten vor. Für den Zeitraum ab Januar 2011 sind alle Informationen zu den Konfliktakteuren, -gegenständen, -maßnahmen und -intensitäten für zwischen-, inner- und substaatliche sowie transnationale Konflikte in allen Weltregionen geografisch disaggregiert erfasst. Der Stand der politischen Konflikte wird vom HIIK in seinem jährlichen Periodikum, dem Konfliktbarometer, publiziert (vgl. HIIK 2012).

Im »War«-Datensatz von COW (Sarkees/Wayman 2010) liegt die Gesamtzahl der erfassten Kriege, die jeweils mindestens zu 1000 konfliktbezogenen Opfern (»battle-related deaths«) je Jahr geführt haben, bei 243 (1946-2007). ${ }^{11}$ Anhand der Konfliktkonstellationen werden neun unterschiedliche Kriegstypen unterschieden, die sich unter die vier Oberkategorien »zwischenstaatliche«, »extrastaatliche«, »innerstaatliche« und »nicht-staatliche Kriege« subsumieren lassen. Die Daten zu ein-

9 In CONIAS sind Konflikte, die Teil des Zweiten Weltkriegs waren, nicht erfasst.

10 Eine ausführliche Darstellung der Daten für den Zeitraum 1945-2005, auch in Verlaufsanalysen, findet sich in Schwank (2012). Die Daten stehen unter www.conias.org zur Verfügung.

11 Die Daten bis zum Ende des Zweiten Weltkriegs wurden nicht berücksichtigt, um eine Vergleichbarkeit zu den anderen Datensätzen herzustellen. 
zelnen Kriegen werden nicht nach Konfliktjahren aufgeschlüsselt, sondern je nach Datensatz nach Konflikten oder Konfliktakteuren. Darüber hinaus sind im »War«Datensatz von COW keine Konfliktmaßnahmen im Sinne eines typologisierten Handelns und Kommunizierens von Konfliktakteuren und keine unterschiedlichen Konfliktintensitäten enthalten.

Der »Militarized Interstate Disputes «-Datensatz (MID) des COW (Jones et al. 1996; Ghosn et al. 2004) umfasst die Drohung, Zurschaustellung oder Nutzung von Gewalt zwischen Staaten, wobei letztere die Kriegsschwelle des »War«-Datensatzes nicht überschreitet. Die Daten sind auf der Konfliktebene aggregiert und umfassen Angaben zu den beteiligten Akteuren, dem Konfliktaustrag, der Dauer des Konflikts, dem Gegenstand des Konflikts sowie zur Konfliktbeendigung. Bezüglich des Konfliktaustrags wird zwischen der Androhung, Zurschaustellung und Nutzung von Gewalt differenziert und jeweils anhand eines Maßnahmenkatalogs genauer spezifiziert. Darüber hinaus werden sieben Intensitätsstufen bezüglich der Anzahl der Todesopfer unterschieden. Insgesamt sind 1524 Dispute (1946-2001) erfasst, die in einem weiteren Datensatz nach beteiligten Akteuren in 3591 Einträge aufgeschlüsselt werden. Im »Dyadic Militarized Interstate Dispute«-Datensatz und dem »Militarized Interstate Incident«-Datensatz, die jeweils die Beobachtungsperiode von 1993 bis 2001 umfassen, werden die Dispute dyadisch aufgeschlüsselt (512 Konfliktdyaden) bzw. einzelne Ereignisse erfasst (2119 Ereignisse).

Das »Armed Conflict Dataset« (ACD) von UCDP/PRIO (Gleditsch et al. 2002; Themnér/Wallensteen 2012) umfasst für den Zeitraum von 1946 bis 2011248 Konflikte bei 2061 Konfliktjahren. Fast drei Viertel (72,6\%) dieser Konfliktjahre sind Konflikte geringer Gewaltintensität (»minor armed conflicts «), gut ein Viertel sind Kriege (27,4\%). Das UCDP »Dyadic Dataset« schlüsselt diese 248 Konflikte in 524 Dyaden auf und umfasst insgesamt 2601 Konfliktdyadenjahre. Im UCDP »NonState Conflict Dataset«, das den Zeitraum von 1989 bis 2011 umfasst, werden 419 nicht-staatliche Konfliktdyaden bei 646 Dyadenjahren erfasst. Die Dyaden werden nicht zu Konflikten mit mehr als zwei Parteien, wie beispielsweise im ACD-Datensatz, zusammengefasst. Ferner werden die Konflikte nicht in niedrig- und hochgewaltsame Konflikte unterschieden, gleichwohl eine solche Differenzierung der Dyadenjahre auf Basis der angegebenen Todesopfer leicht möglich ist. Das UCDP "One-Sided Violence Dataset « erfasst Formen einseitiger staatlicher oder nichtstaatlicher Gewaltanwendung gegen Zivilisten, die zu mindestens 25 Toten im Jahr geführt haben. Insgesamt sind 713 Akteursjahre im Datensatz enthalten.

Der ACD-Datensatz unterscheidet hinsichtlich der Gegenstandsdimension zwei »incompatibilities « (Territorium und Regierung). Diese Inkompatibilitäten sind dabei sowohl notwendige Bedingung der Definition bewaffneter Konflikte, also zwi- 
schenstaatlicher oder innerstaatlicher Konflikte mit Regierungsbeteiligung, als auch Kriterium zur Typenbildung. Im »Non-State Conflict Dataset « und im »One-Sided Violence Dataset« gibt UCDP keine Gegenstandsdimension an, da die möglichen »incompatibilities « nicht zu diesen Konflikttypen passen. Insgesamt umfassen die Datensätze zu organisierter Gewalt 158 staatliche und 996 nicht-staatliche Konfliktakteure, die im UCDP »Actor Dataset« zusammengefasst sind.

Durch die Erfassung einzelner georeferenziell verorteter Konfliktereignisse haben sowohl UCDP als auch PRIO die Datenbasis zur Konfliktanalyse verfeinert. Der GED-Datensatz von UCDP erfasst für bewaffnete Konflikte im subsaharischen und nördlichen Afrika im Zeitraum von 1989 bis 2010 insgesamt 24.381 Konfliktereignisse in 528 unterschiedlichen Dyaden. Dabei entfallen 51\% der erfassten Ereignisse auf Konflikte mit staatlicher Beteiligung, 18\% auf Konflikte ohne staatliche Beteiligung und 31\% auf Fälle einseitiger Gewaltanwendung. Durch die Erfassung von Konfliktjahren, bei denen die Schwelle von 25 Toten pro Jahr unterschritten wurde, werden hierdurch auch Phasen sehr niedriger Gewaltintensität erfasst.

Der ACLED-Datensatz von PRIO unterscheidet acht größtenteils gewaltbezogene Ereignistypen und ordnet diese 40 verschiedenen Konstellationstypen zu (Raleigh et al. 2010). Er umfasst insgesamt über 75.000 Konfliktereignisse für den afrikanischen Kontinent sowie ausgewählte asiatische Länder von 1997 bis 2012 georeferenziell. Dabei entfallen 64.899 der Ereignisse auf den afrikanischen Kontinent.

Tabelle 2: Vergleich unterschiedlicher Konfliktdatenbanken

\begin{tabular}{|c|c|c|c|}
\hline & COW/MID & UCDP/PRIO & CONIAS \\
\hline $\begin{array}{c}\text { Konflikt- } \\
\text { anzahl }\end{array}$ & $\begin{array}{c}\text { 243 Konflikte } \\
\text { (1946-2007) } \\
\text { 1524 Dispute } \\
\text { 512 Disputdyaden } \\
(1993-2001)\end{array}$ & $\begin{array}{c}\text { 248 Konflikte } \\
524 \text { Dyaden } \\
(1946-2011) \\
\text { (1989-2011) }\end{array}$ & $\begin{array}{c}\text { 985 Konflikte } \\
(1946-2012)\end{array}$ \\
\hline $\begin{array}{c}\text { Konflikt- } \\
\text { jahre }\end{array}$ & - & 2061 Konfliktjahre & 15.400 Konfliktjahre \\
\hline $\begin{array}{c}\text { Dyaden- } \\
\text { jahre }\end{array}$ & - & $\begin{array}{c}2601 \text { Dyadenjahre (mit staatl. Bet.) } \\
\text { 646 Dyadenjahre (ohne staatl. } \\
\text { Bet.) }\end{array}$ & 31.800 Dyadenjahre \\
\hline Akteure & - & 1154 Konfliktakteure & ca. 2850 Konfliktakteure \\
\hline $\begin{array}{c}\text { Maßnah- } \\
\text { men }\end{array}$ & $\begin{array}{c}24.381 \text { (UCDP/GED) } \\
(1989-2010)\end{array}$ & ca. 50.000 \\
\hline
\end{tabular}


Auffällig in der Gesamtbetrachtung der Tabelle 2 sind die erheblich voneinander abweichenden Angaben der Datenbanken zur Anzahl der erfassten Konflikte sowie zur erfassten Dauer und den beteiligten Akteuren. In einer aufschlussreichen Studie hatten sich Wolf-Dieter Eberwein und Sven Chojnacki bereits 2001 der bis dahin wenig beachteten Frage gewidmet, wie sich der Unterschied in der Konfliktanzahl zwischen COW, UCDP, AKUF und KOSIMO erklären lässt (Eberwein/Chojnacki 2001). Auch wenn die Autoren damals keine eindeutige Antwort fanden, konnten sie zeigen, dass wider Erwarten Datenbanken mit unterschiedlichen konzeptionellen Ansätzen (quantitativ vs. qualitativ, also beispielsweise AKUF und UCDP sowie KOSIMO und COW) eine höhere Übereinstimmung der Konflikte untereinander aufwiesen als bei einem Datenbankvergleich, bei denen die Paare jeweils den gleichen Ansatz verfolgten (COW mit UCDP und AKUF mit KOSIMO). Eine mögliche Ursache dieses Befundes ist in den jeweiligen Entstehungszeiten der Datenbanken zu suchen und lässt sich damit erklären, dass als Grundlage der neuen Datenbanken die bis dahin jeweils letztmalig aktualisierten etablierten Datenbanken dienten. Für KOSIMO und AKUF war dies COW, für UCDP/PRIO waren dies wiederum KOSIMO und AKUF. So ließ sich der scheinbare Widerspruch auflösen und doch zeigen, dass die verwendete Methode (quantitativ beziehungsweise qualitativ) erheblichen Einfluss auf die Anzahl der Konflikte und der damit verbundenen Attribute hat.

Auch die jeweiligen Zielsetzungen der Datenbanken mögen einen erheblichen Einfluss auf die Anzahl der erfassten Konflikte und deren Dauer haben. Während COW und UCDP jedenfalls anfänglich noch stärker auf das zwischenstaatliche Konfliktschema ausgerichtet waren und es vornehmlich von Belang war, ob in einem bestimmten Land ein gewaltsamer Konflikt zu beobachten war oder nicht, ist CONIAS entwickelt worden, um Konfliktdynamiken zu erfassen (Schwank 2012). Wie bereits am Beispiel der Konflikte in Sri Lanka erläutert, werden deshalb Konflikte, die von außen betrachtet als ein großes komplexes Konfliktgebilde erscheinen, in verschiedene Teilkonflikte untergliedert. Als Folge werden damit auch Konfliktakteure, die bei einer gröberen Betrachtung als unwichtig eingestuft würden, in einer detaillierten Betrachtung als relevant erachtet und somit in den Datensatz aufgenommen. Die größere Anzahl der Konflikte, verbunden mit der genauen Betrachtung der Akteurskonstellationen führt somit letztlich zu einer deutlich höheren Anzahl von Dyadenjahren. 


\subsection{Bewertung}

Eingangs identifizierte der vorliegende Beitrag vier Desiderata der quantitativ-empirischen Konfliktforschung: die Erfassung von gewaltlosen Konflikten und von Gewaltkonflikten unterhalb der Kriegsschwelle, die konzeptionelle Integration unterschiedlicher Konflikttypen in ein einheitliches Modell, die Notwendigkeit einer dynamisierten Betrachtung politischer Konflikte sowie eine mehrdimensionale Operationalisierung von Konfliktintensitäten. Diese Desiderata können als Bewertungskriterien für einen Vergleich der dargestellten Konfliktdatenbanken dienen. Inwiefern können die Datenbanken dazu beitragen, die beobachteten Lücken zu schließen?

Ein Großteil der quantitativen Forschung der letzten zwölf Jahre zu innerstaatlichen Konflikten war auf den Ausbruch und ggf. die Dauer von Bürgerkriegen und anderen Formen bewaffneter Konflikte fokussiert (Schwank et al. 2013). Das von CONIAS verwendete Konzept der Konfliktintensität stellt hingegen auf das gesamte Spektrum des Akteurshandelns ab. Nicht- und niedrig-gewaltsame Konflikte und Konfliktperioden werden, dem ersten identifizierten Desideratum entsprechend, durch die Inklusion nicht-gewaltsamer Konfliktmaßnahmen und hieraus abgeleiteter gewaltloser Intensitätsstufen systematisch erfasst. Demgegenüber erfassen weder der »Armed Conflict«-Datensatz des UCDP noch der »War«-Datensatz des COW Konflikte, die während ihrer gesamten erfassten Laufzeit gewaltlos bleiben oder mit sehr geringem Gewalteinsatz (unterhalb des im ACD-Datensatz verwendeten unteren Schwellenwertes von 25 Konflikttodesopfern pro Jahr) ausgetragen werden. Mit der Ausdehnung der Erfassung auf gewaltfreie Konfliktepisoden vor, zwischen und nach Gewaltphasen und auch auf Zeiträume, in denen nur sehr wenig Gewalteinsatz zu beobachten war, wird durch CONIAS eine größere Bandbreite des Austrags politischer Konflikte erfasst und Analysen zugänglich gemacht. Zudem wird die Abbildung kompletter Konfliktverläufe möglich, so dass Konflikte im Zeitverlauf nicht unterhalb der Kriegsschwelle (COW) oder der Gewaltschwelle (UCDP) »verschwinden«, sondern durchgängig nachgezeichnet werden können. Dies ermöglichen ebenfalls der MID-Datensatz sowie, für einen eingeschränkten Zeitraum, die genannten Ereignisdatensätze. Statt datenbanktechnisch ggf. mehrere Einzelkonflikte zu bilden und in den Auswertungen zu zählen, was die Ursachenanalyse bezüglich des Ausbruchs und der Dauer von Konflikten erheblich verzerren kann, da jeweils mehrere kurze statt ein längerer Konflikt erfasst werden (Ross 2004), verzeichnet CONIAS einen durchgehenden Verlauf des betreffenden Konflikts. Dies bedeutet eine verbesserte Möglichkeit der Analyse von Eskalations-, Deeskalations- und Reeskalationsprozessen (vgl. Gromes 2012). So kann die er- 
neute Eskalation eines über einen längeren Zeitraum gewaltlos fortbestehenden Konflikts vom Ausbruch eines tatsächlich neuen Konflikts unterschieden werden. Weiterhin können Deeskalationsprozesse danach differenziert werden, ob es sich um ein »Auslaufen« des Konflikts handelt, das heißt um ein graduelles Abflauen der Gewalt bei Fortbestand eines gewaltlosen politischen Konflikts, oder um eine klar bestimmbare und effektive Konfliktbeendigung, beispielsweise durch nachhaltige Friedensverträge oder auch durch die Vernichtung eines beteiligten Akteurs.

Der hohe Abstraktionsgrad des Basiskonzepts politischer Konflikte, der hieraus folgende weite Geltungsbereich und die Spezifizierbarkeit der drei deduktiv hergeleiteten konstitutiven Merkmale Akteur, Gegenstand und Maßnahme erlaubt eine systematische Typenbildung für die Gesamtheit der politischen Konflikte (zweites Desideratum). Die CONIAS-Datenbank integriert zwischenstaatliche, innerstaatliche, substaatliche und transnationale Konflikte in einer Datenbank, während sowohl COW als auch UCDP jeweils unterschiedliche Datenbanken mit unterschiedlichen Erfassungszeiträumen umfassen (»Silo-Lösung«). In CONIAS werden auch jene gewaltlosen und gewaltsamen Konflikte erfasst, die nur von nicht-staatlichen Akteuren ausgetragen werden und deshalb im COW-Datensatz ganz fehlen oder bei UCDP gesondert in dyadischer Form erfasst werden. Insbesondere die Konfliktmaßnahmen und -gegenstände werden in CONIAS nicht nur einzeln als solche erfasst, sondern auch kategorial eingeordnet. Mit zehn verschiedenen Konfliktgegenständen und über siebzig Maßnahmenkategorien betrachtet CONIAS das Wollen und Wirken der Konfliktakteure sehr differenziert. Insgesamt gesehen erlaubt der CONIAS-Ansatz eine in hohem Maße ausdifferenzierte und variable Möglichkeit der Typenbildung: Je nach theoretischem Interesse können Konflikttypen in Bezug auf Akteurskonstellationen, Akteurshandeln, Konfliktgegenstände oder Intensitätsniveaus gebildet und auch komplex miteinander kombiniert werden.

Bezogen auf das dritte Desideratum ergibt sich aus diesen beiden genannten Merkmalen - der Inklusivität und Spezifität des Konzepts - die Fähigkeit des Heidelberger Ansatzes zu einer realitätsnahen und methodisch konsistenten Abbildung von Konfliktdynamiken, insbesondere in Bezug auf komplexe Konfliktsituationen. Auch wenn beteiligte Akteure und ihre Konstellation, die Konfliktgegenstände oder die Form des Konfliktaustrags sich wandeln, verbleiben sie innerhalb eines einzigen Konzepts und Datensatzes. Während die COW-Datenbank statisch geblieben ist und allein schon aufgrund der Struktur der Konfliktliste zu einer Fokussierung auf Kriegsausbrüche beiträgt, bietet UCDP mit seinem Angebot, eine Konfliktliste auf Jahresbasis zu erstellen, seiner Unterscheidung zwischen Kriegen und »minor armed conflicts« sowie der Angabe von Todesopferzahlen einen grundsätzlich dynamisierten Zugang zum Konfliktgeschehen. Mit fünf Intensitätsstufen ist die CON- 
IAS-Datenbank in Bezug auf ihre Konzeption jedoch differenzierter. Die unter den Desiderata eins und zwei angesprochene Breite der Konflikterfassung geht daher auch nicht einher mit einer Verwischung bestehender Unterschiede zwischen den mitunter höchst diversen Formen des Konfliktaustrags.

Der CONIAS-Datensatz rekurriert bei der Betrachtung von Gewaltkonflikten nicht, wie sonst fast allgemein üblich, alleinig auf die Erfassung der Zahl der konfliktbedingten Todesopfer, sondern erweitert die Datenerfassung um die Zahl der Flüchtlinge und Vertriebenen, den Umfang des eingesetzten Personals sowie die qualitativen Dimensionen des Waffeneinsatzes und der konfliktbedingten Zerstörung. Im Unterschied zu COW oder UCDP beruht der Heidelberger Ansatz damit auf einer breiteren Basis zur Bestimmung der Konfliktintensität. Die Anzahl der konfliktbezogenen Todesopfer wird nicht aus der Analyse ausgeschlossen, stellt jedoch nur einen Indikator unter mehreren dar und wird an das zielgeleitete Agieren der Konfliktbeteiligten konzeptionell rückgebunden. Die Gesamtschau der Mittel und Folgen gewaltsamer Konfliktmaßnahmen und die Verwendung quantitativer und qualitativer Indikatoren zur Messung von Konfliktintensitäten macht, dem vierten Desideratum entsprechend, die Operationalisierung der Intensitätsstufen valider.

\section{Schlussbetrachtung}

Der vorliegende Beitrag hat den aktuellen Heidelberger Ansatz zur quantitativen Konflikterfassung vorgestellt und konzeptionell und datentechnisch mit den führenden Ansätzen verglichen. Hierzu wurde der Stand der Grundlagenforschung zur Erfassung politischer Konflikte vor dem Hintergrund derzeitig bestehender Desiderata der quantitativ-empirischen Konfliktforschung vergleichend untersucht. Bezüglich der Erfassung von Konflikten unterhalb der Kriegsschwelle sind durch die Inklusion niedrig-gewaltsamer Phasen bei UCDP im Vergleich zu COW bedeutende Fortschritte erzielt worden. CONIAS berücksichtigt darüber hinaus nicht-gewaltsame sowie niedrig-gewaltsame Konflikte und Konfliktphasen unterhalb der von UCDP in Bezug auf Konflikte verwendeten Schwelle von 25 Toten pro Jahr. Die eingangs kritisierte »Silo-Lösung « der Datenerfassung, die separierte Konfliktkonzepte widerspiegelt, findet sich in der quantitativen Forschung immer seltener. COW und UCDP verwenden zwar noch unterschiedliche Datensätze mit unterschiedlichen Erfassungszeiträumen, gewährleisten aber zunehmend die Verknüpfung zwischen diesen. CONIAS strebt durch eine möglichst grundlegende Konzeptionalisierung die Vereinheitlichung seiner Konfliktdaten an, beinhaltet zurzeit jedoch durch die Umstellung auf die geografisch und zeitlich disaggregierte Erfassung 
von Konfliktintensitäten ebenfalls unterschiedliche Datensätze. Eine dynamisierte Betrachtung von Konflikten ist im Rahmen der COW-Datenbank unter Berücksichtigung der MID-Daten eingeschränkt möglich. Die Differenzierung unterschiedlicher Intensitätsstufen und Konfliktkonstellationen im Zeitverlauf - in zwei Stufen beim ACD-Datensatz des UCDP und in fünf Stufen bei CONIAS - erlaubt eine differenziertere Analyse von Verlaufsformen. Eine mehrdimensionale Intensitätsbestimmung über die Betrachtung von konfliktbedingten Todesopfern hinaus ist ein Alleinstellungsmerkmal der CONIAS-Datenbank.

Es hat sich gezeigt, dass UCDP und PRIO Stärken vor allen Dingen in Bereichen aufweisen, in die CONIAS aufgrund der Breite seines Ansatzes bislang nicht vorstoßen konnte, nämlich in der umfangreichen Erfassung georeferenziell codierter Konfliktereignisse. Demgegenüber nimmt CONIAS mit seinen soeben identifizierten charakteristischen Merkmalen einen anderen Blickwinkel ein und erhellt Bereiche des Konfliktgeschehens, die bislang wenig erforscht wurden. In der Gesamtschau ermöglichen es die zeitgenössischen quantitativ-vergleichend orientierten Konfliktkonzepte und Datensätze, ein realistischeres Bild des empirischen Konfliktgeschehens zu zeichnen. 


\section{Literatur}

Billing, Peter 1992: Eskalation und Deeskalation internationaler Konflikte: Ein Konfliktmodell auf der Grundlage der empirischen Auswertung von 288 internationalen Konflikten seit 1945, Frankfurt a. M.

Bremer, Stuart A. 1993: Advancing the Scientific Study of War, in: International Interactions 19: 1-2, 1-26.

Brzoska, Michael 2004: >New Wars〈 Discourse in Germany, in: Journal of Peace Research 41: 1, 107-117.

Buhaug, Halvard/Lujala, Päivi 2005: Accounting for Scale: Measuring Geography in Quantitative Studies of Civil War, in: Political Geography 24: 4, 399-418.

Chojnacki, Sven 2004: Wandel der Kriegsformen? - Ein kritischer Literaturbericht, in: Leviathan 32: 3, 402-424.

Chojnacki, Sven 2006: Kriege im Wandel. Eine typologische und empirische Bestandsaufnahme, in: Geis, Anna (Hrsg.): Den Krieg überdenken. Kriegsbegriffe und Kriegstheorien in der Kontroverse, Baden-Baden, 47-74.

Croicu, Mihai Catalin/Sundberg, Ralph 2012: UCDP GED Conflict Polygons Dataset Codebook. Version 1.1, in: http://www.ucdp.uu.se/ged/data/ucdp-ged-poly gons-v-1-1-codebook.pdf; 7.3.2013.

Croissant, Aurel/Wagschal, Uwe/Schwank, Nicolas/Trinn, Christoph 2009: Kulturelle Konflikte seit 1945. Die kulturellen Dimensionen des globalen Konfliktgeschehens, Baden-Baden.

Cunningham, David E./Gleditsch, Kristian Skrede/Salehyan, Idean 2009: It Takes Two. A Dyadic Analysis of Civil War Duration and Outcome, in: Journal of Conflict Resolution 53: 4, 570-597.

DeRouen, Karl R./Sobek, David 2004: The Dynamics of Civil War Duration and Outcome, in: Journal of Peace Research 41: 3, 303-320.

DeRouen, Karl R./Bercovitch, Jacob 2008: Enduring Internal Rivalries: A New Framework for the Study of Civil War, in: Journal of Peace Research 45: 1, 55-74.

Dixon, Jeffrey 2009: What Causes Civil Wars? Integrating Quantitative Research Findings, in: International Studies Review 11: 4, 707-735.

Eberwein, Wolf-Dieter/Chojnacki, Sven 2001: Scientific Necessity and Political Utility: A Comparison of Data on Violent Conflicts, in: https://www.econstor. eu/dspace/bitstream/10419/49845/1/339880880.pdf; 7.3.2013.

Eck, Kristine 2005: A Beginner's Guide to Conflict Data. Finding and Using the Right Dataset, in: http://www.pcr.uu.se/digitalAssets/18/18128_UCDP_paper1. pdf; 7.3.2013. 
Fjelde, Hanne/Nilsson, Desirée 2012: Rebels against Rebels. Explaining Violence between Rebel Groups, in: Journal of Conflict Resolution 56: 4, 604-628.

Fürnkranz, Johannes/Petrak, Johann/Trappl, Robert 1997: Knowledge Discovery in International Conflict Databases, in: Applied Artificial Intelligence 11: 1, 91-118.

Gantzel, Klaus Jürgen 1986: Die Kriege nach dem Zweiten Weltkrieg bis 1984 : Daten und erste Analysen, München.

Gantzel, Klaus Jürgen 1988: Krieg in der Dritten Welt als Forschungsgegenstand. Eine Einführung in das Thema, in: ders. (Hrsg): Krieg in der Dritten Welt: Theoretische und methodische Probleme der Kriegsursachenforschung - Fallstudien, Baden-Baden, 25-81.

Ghosn, Faten/Palmer, Glenn/Bremer Stuart A. 2004: The MID3 Data Set, 1993-2001: Procedures, Coding Rules, and Description, in: Conflict Management and Peace Science 21: 2, 133-154.

Glasl, Friedrich 2002: Konfliktmanagement. Ein Handbuch für Führungskräfte, Beraterinnen und Berater, Stuttgart.

Gleditsch, Nils Petter/Wallensteen, Peter/Eriksson, Mikael/Sollenberg, Margareta/ Strand, Håvard 2002: Armed Conflict 1946-2001: A New Dataset, in: Journal of Peace Research 39: 5, 615-637.

Gromes, Thorsten 2012: Der Rückfall in den Bürgerkrieg, in: Zeitschrift für Friedens- und Konfliktforschung 1: 2, 275-305.

Hasenclever, Andreas 2002: Sie bewegt sich doch. Neue Erkenntnisse und Trends in der quantitativen Kriegsursachenforschung, in: Zeitschrift für Internationale Beziehungen 9: 2, 331-364.

Hegre, Håvard/Sambanis, Nicholas 2006: Sensitivity Analysis of Empirical Results on Civil War Onset, in: Journal of Conflict Resolution 50: 4, 508-535.

HIIK - Heidelberger Institut für Internationale Konfliktforschung (Hrsg.) 2012: Conflict Barometer 2011. Disputes, Non-violent Crises, Violent Crises, Limited Wars, Wars, Heidelberg.

Jones, Daniel M./Bremer, Stuart A./Singer, J. David 1996: Militarized Interstate Disputes, 1816-1992: Rationale, Coding Rules, and Empirical Patterns, in: Conflict Management and Peace Science 15: 2, 163-213.

Kaldor, Mary 1999: New and Old Wars: Organized Violence in a Global Era, Stanford, CA.

Kende, Istvan 1971: Twenty-Five Years of Local Wars, in: Journal of Peace Research 8: 1, 5-22.

Kende, Istvan 1982: Kriege nach 1945. Eine empirische Untersuchung, Frankfurt a. M. 
Korf, Benedikt 2011: Resources, Violence and the Telluric Geographies of Small Wars, in: Progress in Human Geography 35: 6, 733-756.

Mello, Patrick A. 2010: In Search of New Wars: The Debate about a Transformation of War, in: European Journal of International Relations 16: 2, 297-309.

Most, Benjamin A./Starr, Harvey 1983: Conceptualizing »War«. Consequences for Theory and Research, in: Journal of Conflict Resolution 27: 1, 137-159.

Münkler, Herfried 2002: Die neuen Kriege, Reinbek bei Hamburg.

Ó Tuathail, Gearóid 2010: Localizing Geopolitics. Disaggregating Violence and Return in Conflict Regions, in: Political Geography 29: 5, 256-265.

Pavlovic, Nada J./Casagrande Hoshino, Lisa/Mandel, David R./Dorn, Walter A. 2008: Indicators and Indices of Conflict and Security. A Review and Classification of Open-Source Data. Technical Report, Toronto.

Pettersson, Therése/Themnér, Lotta 2012: UCDP Actor Dataset Codebook, http:// www.pcr.uu.se/research/ucdp/datasets/ucdp_actor_dataset/; 2.9.2012.

Pfetsch, Frank R. 1991 a: Nationale und internationale Konflikte nach 1945, in: Politische Vierteljahresschrift 32: 2, 258-285.

Pfetsch, Frank R. (Hrsg.) 1991 b: Konflikte seit 1945. Daten - Fakten - Hintergründe, Freiburg.

Pfetsch, Frank R./Billing, Peter 1994: Datenhandbuch nationaler und internationaler Konflikte, Baden-Baden.

Pfetsch, Frank R./Rohloff, Christoph 2000 a: National and International Conflicts, 1945-1995. New Empirical and Theoretical Approaches, London.

Pfetsch, Frank R./Rohloff, Christoph 2000 b: KOSIMO. A Databank on Political Conflict, in: Journal of Peace Research 37: 3, 379-389.

Raleigh, Clionadh 2010: Empirical and Theoretical Research in International Relations, in: International Interactions 36: 4, 384-410.

Raleigh, Clionadh/Linke, Andrew/Hegre, Håvard/Karlsen, Joakim 2010: Introducing ACLED. An Armed Conflict Location and Event Dataset, in: Journal of Peace Research 47: 5, 651-660.

Ross, Michael L. 2004: What Do We Know about Natural Resources and Civil War?, in: Journal of Peace Research 41: 3, 337-356.

Salehyan, Idean/Gleditsch, Kristian Skrede 2006: Refugee Flows and the Spread of Civil War, in: International Organization 60: 2, 335-366.

Salehyan, Idean/Gleditsch, Kristian Skrede/Cunningham, David E. 2011: Explaining External Support for Insurgent Groups, in: International Organization 65: 4, 709-744. 
Sambanis, Nicholas 2002: A Review of Recent Advances and Future Directions in the Quantitative Literature on Civil Wars, in: Defence and Peace Economics 13: 3, 215-243.

Sambanis, Nicholas 2004: What is Civil War?, in: Journal of Conflict Resolution 48: 6, 814-858.

Sarkees, Meredith Reid/Wayman, Frank 2010: Resort to War. 1816-2007, Washington, DC.

Schlichte, Klaus 2002: Neues über den Krieg? Einige Anmerkungen zum Stand der Kriegsforschung in den Internationalen Beziehungen, in: Zeitschrift für Internationale Beziehungen 9: 1, 113-137.

Schlichte, Klaus 2006: Neue Kriege oder alte Thesen? Wirklichkeit und Repräsentanten kriegerischer Gewalt in der Politikwissenschaft, in: Geis, Anna (Hrsg.): Den Krieg überdenken, Frankfurt a. M., 111-131.

Schlichte, Klaus 2009: In the Shadow of Violence. The Politics of Armed Groups, Frankfurt a. M.

Schwank, Nicolas 2012: Konflikte, Krisen, Kriege. Eskalationsrisiken politischer Konflikte seit 1945, Baden-Baden.

Schwank, Nicolas/Trinn, Christoph 2010: Muster und Entwicklungstrends politischer Konflikte im Spiegel des Conflict Information System (CONIS) Heidelberg, in: Feichtinger, Walter/Dengg, Anton (Hrsg.): Kein Feind in Sicht. Konfliktbilder und Bedrohungen der Zukunft, Wien, 65-87.

Schwank, Nicolas/Trinn, Christoph/Wencker, Thomas 2013: The Causes of Violent Escalation of Intrastate Conflicts, i. E.

Schwank, Nicolas/Wencker, Thomas/Trinn, Christoph 2012: Méthodologie de la recherche sur les conflits à Heidelberg, in: Diplomatie, le Grands Dossiers 7/2012, 26-29.

Seybolt, Taylor B. 2002: Measuring Violence. An Introduction to Conflict Data Sets, in: S.I.P.R. Institute (Hrsg.): SIPRI Yearbook 2002, Oxford, 81-96.

Singer, David J./Small, Melvin 1972: The Wages of War 1816-1965. A Statistical Handbook, New York, NY.

Sundberg, Ralph/Lindgren, Mathilda/Padskocimaite, Ausra 2011: UCDP Georeferenced Event Dataset (GED) Codebook, Version 1.0., Uppsala.

Sundberg, Ralph/Eck, Kristine/Kreutz, Joakim 2012: Introducing the UCDP NonState Conflict Dataset, in: Journal of Peace Research 49: 2, 351-362.

Themnér, Lotta/Wallensteen, Peter 2012: Armed Conflict, 1946-2011, in: Journal of Peace Research 47: 4, 565-575. 
Wagschal, Uwe/Croissant, Aurel/Metz, Thomas/Schwank, Nicolas/Trinn, Christoph 2010: Kulturkonflikte in inner- und zwischenstaatlicher Perspektive, in: Zeitschrift für Internationale Beziehungen 17: 1, 5-37.

Weidmann, Nils B. 2009: Geography as Motivation and Opportunity, in: Journal of Conflict Resolution 53: 4, 526-543.

Wright, Quincy 1942: A Study of War, Chicago, IL.

\section{Die Autoren}

Dr. Nicolas Schwank ist Leiter der CONIAS-Forschungsgruppe.

E-Mail:schwank@conis.org

Christoph Trinn, M.A. ist Mitglied des Promotionskollegs »Politikperformanz autokratischer und demokratischer Regime « des Instituts für Politische Wissenschaft der Universität Heidelberg und Vorstandsmitglied der CONIAS-Forschungsgruppe.

E-Mail: christoph.trinn@ipw.uni-heidelberg.de

Thomas Wencker, M.A. ist wissenschaftlicher Mitarbeiter des Instituts für Politische Wissenschaft der Universität Heidelberg und Vorstandsmitglied der CONIAS-Forschungsgruppe.

E-Mail: thomas.wencker@ipw.uni-heidelberg.de 